\title{
Calibration of Dynamic Molecular Rulers Based on Plasmon Coupling between Gold Nanoparticles
}

\author{
Björn M. Reinhard, Merek Siu, Harish Agarwal, \\ A. Paul Alivisatos and Jan T. Liphardt
}

\section{Supporting Information}

\section{Materials and Methods}

General Procedures: Aqueous solutions of 40 and $80 \mathrm{~nm}$ gold nanoparticles (British Biocell International, BBI) with a concentration of $9.0 \times 10^{10}$ particles $/ \mathrm{mL} \approx 1.5 \times 10^{-10} \mathrm{M}$ and $1.1 \times 10^{10}$ particles $/ \mathrm{mL} \approx 1.8 \mathrm{x}$ $10^{-11} \mathrm{M}$ respectively were purchased from Ted Pella. Deionized water (> 18.0 M $\Omega$ ) from a Millipore MilliQ Academic water purification system was used for all experiments. All buffers were filtered with a $0.2 \mu \mathrm{m}$ pore-size GH polypro (GHP) membrane microfilter (Pall) prior to use. Immediately after conjugation, the particles were immersed in storage buffer, Superblock (Pierce 37515), and stored at 4 degree Celsius for up to one month.

Preparation of Neutravidin Nanoparticle Conjugates: Neutravidin (Molecular Probes, A2666) was dissolved in buffer1 (10 mM Tris, $\mathrm{pH} 7.0$ and $50 \mathrm{mM} \mathrm{NaCl})$ at a concentration of $1 \mathrm{mg} / \mathrm{mL} .250 \mu \mathrm{L}$ of this Neutravidin solution was added to $10 \mathrm{~mL}$ of aqueous nanoparticle solution. After mixing thoroughly, 100 $\mu \mathrm{L}$ of $1.0 \mathrm{M} \mathrm{NaHCO}_{3}$ was added to the reaction mix, which was then allowed to react for 10 minutes. Subsequently $50 \mu \mathrm{L}$ of an aqueous solution containing $2 \%$ polyethylene glycol 6000 (Merck, 807491) was added and the reaction mixture was centrifuged at $800 \mathrm{G}$ for 35 minutes for $40 \mathrm{~nm}$ particles or 10 minutes for $80 \mathrm{~nm}$ particles. The pellets were washed with $1 \mathrm{~mL}$ buffer $2\left(980 \mu \mathrm{L} \mathrm{H}_{2} \mathrm{O}, 10 \mu \mathrm{L} 1.0 \mathrm{M} \mathrm{NaHCO}_{3}, 10\right.$ $\mu \mathrm{L} 2 \%$ polyethylene glycol 6000) and centrifuged again at $800 \mathrm{G}$ for $35 \mathrm{~min}$ for $40 \mathrm{~nm}$ particles or $10 \mathrm{~min}$ for $80 \mathrm{~nm}$ particles. Finally the pellets were resuspended in Superblock and stored at $4{ }^{\circ} \mathrm{C}$.

Preparation of Anti-Digoxigenin Nanoparticle Conjugates: Anti-digoxigenin (Roche Applied Science, 11333089001) was dissolved in buffer1 at a concentration of $1 \mathrm{mg} / \mathrm{mL}$. This solution was dialysed overnight (Pierce 69550). $100 \mu \mathrm{L}$ of the dialysed solution was added to $1 \mathrm{~mL}$ of aqueous nanoparticle solution. After mixing thoroughly, $10 \mu \mathrm{L}$ of $1.0 \mathrm{M} \mathrm{NaHCO}_{3}$ was added to the reaction mix, which was then allowed to react for between 10 minutes and 2 hours. Subsequently the reaction mixture was processed like the Neutravidin nanoparticle conjugates. 
Surface Immobilization of Nanoparticle Dimers: All dimer assemblies werre conducted within a hollow rectangular glass capillary (Vitrocom W5010-100) that served as flowchamber. The chamber had the dimensions (pathlength $\mathrm{x}$ width $\mathrm{x}$ length) $0.1 \mathrm{~mm} \times 1.00 \mathrm{~mm} \times 100 \mathrm{~mm}$ and a wall thickness of $0.1 \mathrm{~mm}$. Biotin-BSA (Sigma, A8549) was dissolved in buffer1 to a total concentration of $1 \mathrm{mg} / \mathrm{mL}$, and $100 \mu \mathrm{L}$ was flowed into the chamber and allowed to incubate for 15 minutes after which the chamber was washed with $200 \mu \mathrm{L}$ of buffer1. Then $50 \mu \mathrm{L}$ of a 1:1000 dilution of nanoparticle conjugate of Neutravidin in buffer 1 was added to the chamber and incubated for two minutes. Next, the chamber was washed with $300 \mu \mathrm{L}$ buffer1. Subsequently dsDNA functionalized with biotin and anti-Dig at opposing ends, was incubated in the chamber for $1 \mathrm{~h}$. The concentration of dsDNA was $40 \mathrm{nM}$ in buffer1. Oligonucleotides with the following sequences were obtained from IDT (10mer, 20mer, 40mer, 250mer) or Fidelity Systems (67mer, 110mer):

for 10 bps DNA

SeqA:

[Biotin]-GCGCTCATCG

SeqA_complement:

[Digoxigenin]-CGATGAGCGC

for 20 bps DNA

SeqB:

[Biotin]-CAGTGACTATGCAGTGCTCC

SeqB_complement:

[Digoxigenin]-GGAGCACTGCATAGTCACTG

for 40 bps DNA

SeqC:

[Biotin]-CAGTGACTATGCAGTGCTCCTAGGTCTATATGCGTTGATG

SeqC_complement:

[Digoxigenin]-CAACGCATATAGACCTAGGAGCACTGCATAGTCACTG

for 67 bps DNA

SeqD:

CTGCCGTGGATCATGTTGCACCAAAATAGTGCTTTAATGTTAACTTAAGCACCACATTGGTGC AACAGATCTC-[Biotin]-T

SeqD_complement:

GAGATCTGTTGCACCAATGTGGTGCTTAATGTTAACATTAAAGCACTATTTTGGTGCAACATG ATCACACGGCAG-[Digoxigenin]

for 110 bps DNA

SeqE

[Biotin]CTTTCTTCTTTGCCTCGAGAAACATAAAAAATTTATTTGCTTTCAGGAAAATTTTTCTGT ATAATAGATTCATAAATTTGAGAGAGGAGTTTAAATATGGCTGGTTCTCG

SeqE_complement:

[Digoxigenin]CTGCCGTGCGAGAACCAGCCATATTTAAACTCCTCTCTCAAATTTATGAATCTAT

TATACAGAAAAATTTTCCTGAAAGCAAATAAATTTTTTATGTTTCTCGAGGCAAAGAAGAAAG

for 250 bps DNA

The following biotin and digoxigenin labeled primers were used to PCR amplify a 250bp fragment from E. coli genomic DNA (kind gift from Sydney Kustu)

5' - [Digoxigenin]-CTTTGTGATCGCTTTCACGGAGCA

5' - [Biotin]-TGGTCGTCGTGGTAACGAAATCTG 
After incubating the DNA, the chamber was washed with buffer1. Then $0.5 \mu \mathrm{L}$ anti-digoxigenin modified gold particles in $50 \mu \mathrm{L}$ buffer 1 were incubated in the chamber for $10 \mathrm{~min}$. After that the chamber was flushed again with buffer.

Experimental Measurements of Plasmon Shift vs. Interparticle Spacing. Directed assembly of the plasmon rulers was followed in real-time with a darkfield microscope; upon formation of a dimer, the intensity of the scattered light increased (Fig. 1c) and the spectrum of the light scattered off the particles was redshifted (Fig. 1d). Subsequently, we recorded spectra of all the particles in the field of view that indicated dimer formation by a change of color and intensity. All spectra reported here were taken at $2 \mathrm{~Hz}$ but due to the larger scattering cross section a better time resolution is feasible for the $80 \mathrm{~nm}$ plasmon ruler (in our set up $\sim 10 \mathrm{~Hz}$ ). All spectra were background and whitelight corrected. For a given dimer, the peak wavelengths from 20 frames were averaged to give the plasmon resonance for a particular dimer. Approximately 30 dimer pairs were measured for each spacer length.

Experimental Setup: The darkfield optical setup is based on a Zeiss Axioplan2. The setup included a 100 W tungsten lamp, an oil immersion darkfield condenser (numerical aperture between 1.3 and 1.6) and a 40x objective with a numerical aperture of 0.65 and a working distance of $0.3 \mathrm{~mm}$. The scattered light was analyzed by a Peltier cooled CCD camera (Spec-10, Roper Scientific) attached to an imaging spectrometer (Acton Research, SP-150 with a 300 grooves per mm grating).

Simulations: We used the discrete dipole approximation (DDA) ${ }^{1}$ and the T-matrix method ${ }^{2}$ to simulate the scattering spectra of single and of pairs of gold nanoparticles. The DDA relies on dividing an object into numerous small volume elements, each with a polarizability given by the refractive index ratio of the volume element to its local environment, and solving the electric field in each volume element selfconsistently. We computed the refractive index of the gold nanoparticles from the bulk dielectric function described by Johnson and Christy. The orientational averaging was done numerically; a separate DDA calculation was performed for every incident light direction. The T-matrix approach expands the fields within and outside an object in spherical vector harmonics (with a multipole cutoff set by the desired accuracy). The fields are then solved self-consistently using a transition matrix to relate the incident field to the scattered field, and boundary conditions (dependent on geometry and the refractive index ratio of the object to its environment) to relate the internal fields to the external fields. The T-matrix approach lends itself to analytical orientational averaging in situations with axial symmetry. Although the transition matrix is in general difficult to solve for arbitrary shapes, it is well known for spherical particles from Mie theory. While the DDA method quickly becomes computationally complex with increasing accuracy (more volume elements and more incident, scattered wavevector pairs), the T-matrix approach converges upon a solution rapidly without having to use many high order vector harmonics. 


\section{Determination of the DNA coverage of Neutravidin gold nanoparticle conjugates}

Neutravidin coated gold nanoparticles were incubated in a solution of biotinylated DNA with a fluorescent tag (Cy5) in a total volume of $100 \mu \mathrm{L}$. The concentration of gold nanoparticles was $\sim 8 \mathrm{pM}$, the ratio of nanoparticles: DNA was approximately 1:5000. After $1 \mathrm{~h}$ the volume was brought to $1 \mathrm{~mL}$ with buffer $\left(\mathrm{H}_{2} \mathrm{O} / \mathrm{NaHCO}_{3} /\right.$ polyethylene glycol 6000) and the solution was centrifuged at $1200 \mathrm{G}$ for $1 \mathrm{~h}$. The pellet was resuspended in buffer and washed three times. After the last wash the pellet was resuspended in $30 \mu \mathrm{L}$ formamide and incubated at $94{ }^{\circ} \mathrm{C}$ for $5 \mathrm{~min}$ to denature the Neutravin-biotin bond. For oligonucleotides immobilized on streptavidin coated magnetic beads the recovery efficiency with formamide is known to be $>90 \%{ }^{3}$

After the solution had cooled down to room temperature, the solution was centrifuged at $14000 \mathrm{G}$ for $20 \mathrm{~min}$. The supernatant was removed and analyzed by gel electrophoresis (15\% polyacrylamide gel containing urea, Figure S1a). Through excitation at $532 \mathrm{~nm}$ the in-gel fluorescence intensity of the recovered DNA was measured on a gel scanner (Amersham Biosciences Typhoon 8600). The concentration was determined by comparison with the fluorescence intensity of known concentrations of the same Cy5 labeled DNA.

a

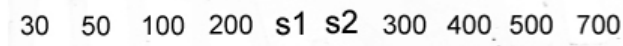

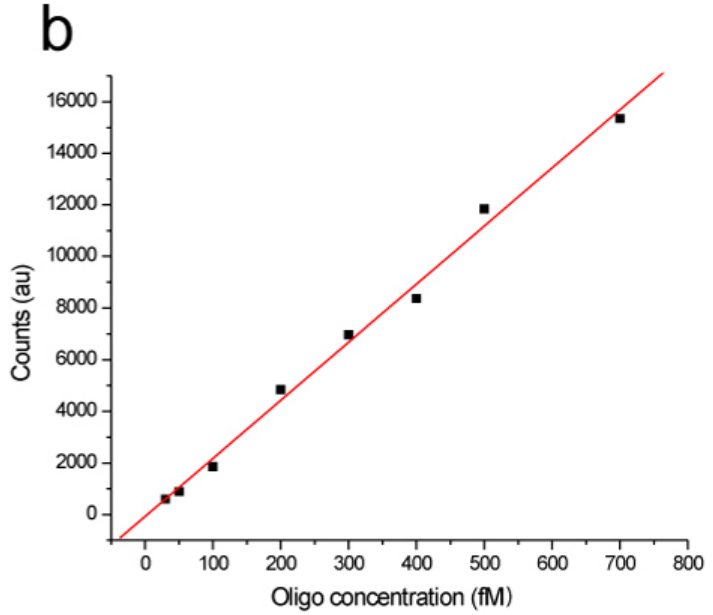

Figure S1 Quantification of DNA bound to $40 \mathrm{~nm}$ Neutravidin coated gold nanoparticles. (a) 15\% TBE urea gel containing Cy5 labeled DNA at concentrations of 13, 40, 100, 200, 300, 400, 500, $700 \mathrm{fM}$ and two samples (s1 and s2) of recovered DNA (see text). (b) Measured fluorescence intensity versus DNA concentration relationship for the conversion of the measured fluorescent intensities of the recovered DNA into concentrations.

(1) Draine, B. T.; Flatau, P. J. J. Opt. Soc. Am. A 1994, 11, 1491-1499.

(2) Mishchenko, M. I.; Travis, L. D.; Lacis, A. A. Scattering, Absorption, and Emission of Light by Small Particles; Cambridge University Press: Cambridge, 2002.

(3) Bai, X. P.; Kim, S. B.; Li, Z. M.; Turro, N. J.; Ju, J. Y. Nucleic Acids Research 2004, 32, 535-541. 